\title{
New Massive Gravity on de Sitter Space and Black Holes at the Special Point
}

\author{
Gregory Gabadadze, ${ }^{1}$ Gaston Giribet, ${ }^{2}$ and Alberto Iglesias ${ }^{1}$ \\ ${ }^{1}$ Center for Cosmology and Particle Physics, New York University \\ 4 Washington Place, New York, NY 10003 USA \\ ${ }^{2}$ Physics Department, University of Buenos Aires and IFIBA-CONICET \\ Ciudad Universitaria, Pabellón 1, 1428, Buenos Aires, Argentina
}

\begin{abstract}
We study New Massive Gravity on de Sitter background and discuss several interrelated properties: The appearance of an enhanced symmetry point at linearized level where the theory becomes partially massless; its absence at full nonlinear level, and its relation with the existence of static black hole solutions and their hair parameter.
\end{abstract}

PACS numbers:

\section{INTRODUCTION}

A fully nonlinear covariant theory of massive gravity in $2+1$ dimensional spacetime, dubbed New Massive Gravity (NMG), has been introduced in Ref. [1]. At the linearized level, the theory is equivalent to the Fierz-Pauli action (FP) for a massive spin-2 field. It also passes highly nontrivial consistency checks at the nonlinear level[2, 3].

In this note, we revisit the formulation of NMG on de Sitter (dS) spacetime. We analyze the theory linearized about dS and study the appearance of an enhanced symmetry at a special point of the parameter space, similarly as it happens in FP gravity on dS [4 7]. This symmetry enhancement has also been observed in Ref. [8] within the canonical approach; here, we go further by relating this symmetry with the existence of dS black holes at the very special point of the parameter space.

\section{NEW MASSIVE GRAVITY ON DE SITTER SPACE}

The action of NMG is given by [1]

$$
S=\frac{1}{16 \pi G} \int d^{3} x \sqrt{-g}\left[R-2 \lambda-\frac{1}{m^{2}} K\right], \quad K=R_{\mu \nu} R^{\mu \nu}-\frac{3}{8} R^{2} .
$$

The square-curvature terms $K$ satisfy the remarkable property $g^{\mu \nu} \delta K / \delta g^{\mu \nu}=K$, which is important for the unitarity of the theory about flat background.

The equations of motion derived from (1) admit dS spacetime as exact solution provided $\lambda \leq m^{2}$, with the Hubble constant $H^{2}=2 m^{2}\left(1 \pm \sqrt{1-\lambda m^{-2}}\right)$.

We consider perturbations of the form $g_{\mu \nu}=\gamma_{\mu \nu}+h_{\mu \nu}$, where $\gamma_{\mu \nu}$ denotes the metric of $\mathrm{dS}_{3}$ space. We denote the covariant derivative with respect to the background metric by $\nabla$ and $\nabla_{\mu} \gamma^{\mu \nu} \nabla_{\nu}$ by $\square$. It is possible to choose transverse traceless gauge $\nabla_{\rho} h_{\mu}^{\rho}=0, h=\gamma^{\mu \nu} h_{\mu \nu}=0$, and the equations of motion read

$$
-\frac{1}{2} \square h_{\mu \nu}+\lambda h_{\mu \nu}-\frac{1}{2 m^{2}}\left(-\square^{2} h_{\mu \nu}+2 H^{2} \square h_{\mu \nu}+\frac{5}{2} H^{2} \square h_{\mu \nu}-\frac{11}{2} H^{4} h_{\mu \nu}\right)=0,
$$

where we used that $\nabla^{\rho} \nabla_{\mu} h_{\nu \rho}=\nabla_{\mu} \nabla^{\rho} h_{\nu \rho}+3 H^{2} h_{\mu \nu}-H^{2} \gamma_{\mu \nu} h$. Interestingly, the equations (2) factorize into

$$
\left(\square-m^{2}-\frac{5}{2} H^{2}\right)\left(\square-2 H^{2}\right) h_{\mu \nu}=0 .
$$

This splits the space of solutions into two: the General Relativity modes, $h_{\mu \nu}$, that solve equation $\left(\square-2 H^{2}\right) h_{\mu \nu}=0$, and the massive mode, $\tilde{h}_{\mu \nu}$, that solves $\left(\square-m^{2}-5 H^{2} / 2\right) \tilde{h}_{\mu \nu}=0$. In the limit $m \rightarrow \infty$, the latter decouples.

\section{CONFORMAL SYMMETRY AT THE SPECIAL POINT}

Here, we will be concerned with the special points $\lambda=m^{2}$, that is $H^{2}=2 m^{2}$. At this point, the theory exhibits a special property. To see this, consider the variation of action (11) under the transformation

$$
\delta g_{\mu \nu}=\gamma_{\mu \nu} \phi
$$


for an arbitrary function $\phi$. Up to a total derivative, the variation of the action is

$$
\delta S=\int d^{3} x \sqrt{-g}\left[\frac{1}{2}\left(1-\frac{H^{2}}{2 m^{2}}\right)\left(\square h-\nabla_{\rho} \nabla_{\sigma} h^{\rho \sigma}\right)+\left(\lambda-\frac{H^{4}}{4 m^{2}}\right) h\right] \phi .
$$

We see that, if $\lambda=m^{2}$ (equivalently, $H^{2}=2 m^{2}$ ), the variation does vanish for arbitrary $\phi$; therefore, at this point the linarized theory exhibits a symmetry enhancement. This is reminiscent of the special point of FP gravity on $\mathrm{dS}$. In the case of FP theory, the enhanced symmetry is of the form $\delta g_{\mu \nu}=\left(H^{-2} \nabla_{\mu} \nabla_{\nu}+\gamma_{\mu \nu}\right) \phi$. NMG is generally covariant, so that the term $\nabla_{\mu} \nabla_{\nu} \phi$ represents a symmetry by itself; it is the $\gamma_{\mu \nu} \phi$ term that becomes enhanced at $\lambda=m^{2}$.

\section{BLACK HOLES AT THE SPECIAL POINT}

It turns out that, at $\lambda=m^{2}$, NMG exhibits another peculiar property: it admits dS black hole solutions [9, 10]. The metrics is

$$
d s^{2}=-\left(-(H r)^{2}+2 b H r-\mu\right) d t^{2}+\frac{d r^{2}}{-(H r)^{2}+2 b H r-\mu}+r^{2} d \theta^{2}
$$

where $b$ and $\mu$ are two arbitrary parameters. In the range $0<\mu \leq b^{2}$, metric (6) describes a black hole that asymptotes $\mathrm{dS}_{3}$ spacetime. The black hole exhibits a curvature singularity at the origin, it being covered by an event horizon located at $r_{-}=(b / H)\left(1-\sqrt{1-\mu / b^{2}}\right)$. The cosmological horizon of $\mathrm{dS}_{3}$ space is located at $r_{+}=(b / H)\left(1+\sqrt{1-\mu / b^{2}}\right)$.

Considering $b$ as small parameter and expanding to linear order one gets

$$
d s^{2} \approx g_{\mu \nu}^{0} d x^{\mu} d x^{\nu}-2 b H r d t^{2}-\frac{2 b H r}{\left(\mu+(H r)^{2}\right)^{2}} d r^{2},
$$

where $g_{\mu \nu}^{0}$ corresponds to metric (7) with $b=0$. Now, we observe that a special combination of a conformal symmetry and coordinate transformations of the form

$$
\delta g_{\mu \nu}=\nabla_{(\mu} \xi_{\nu)}+g_{\mu \nu}^{0} \phi, \quad \text { with } \xi^{\nu}=-\frac{b H r^{2}}{\mu} \delta_{r}^{\nu}, \quad \phi=\frac{2 b H r}{\mu},
$$

precisely cancels the terms proportional to $b$ in (7). This means that (7) can be generated by a transformation (4). At the nonlinear level, the symmetry (4) is absent.

Away from the special point, the linear analysis of NMG reveals the existence of a non-propagating tensor mode of 3D massless General Relativity, as well as the helicity-2, helicity-1, and the helicity-0 modes of a 3D massive graviton (helicity-2 being nondynamical). In contrast, at the special point, the kinetic term of the helicity-0 mode vanishes, and it becomes infinitely strongly coupled since the corresponding conformal symmetry is present at the linear level only. These facts get reflected onto the black hole solution (6) as follows: as shown above, the black hole hair is a gauge artifact in the linearized theory, while this hair is not removable in the full nonlinear case ${ }^{1}$.

It appears that the consistency with no-hair theorems prevents the existence of the black holes away from the special point since for $\lambda \neq m^{2}$ the extra longitudinal mode, which would provide the hair, is a propagating field. At $\lambda=m^{2}$ the black holes are possible only in the regime where the hair is carried by the longitudinal mode that is very (or perhaps infinitely) strongly coupled, at least when the $\mathrm{dS}$ horizon is approached. This feature is what seems to be responsible for the evasion of the no-hair theorems. It may be interesting to study similar questions in 3D ghost-free massive gravity [11, 12]. The latter theory differs from NMG by the absence in it of the massless 3D GR field. This field is responsible for negative mass, $-\mu$ in (6).

[1] E. A. Bergshoeff, O. Hohm and P. K. Townsend, Phys. Rev. Lett. 102, 201301 (2009).

[2] S. Deser, Phys. Rev. Lett. 103, 101302 (2009).

\footnotetext{
${ }^{1}$ Note, however, that on the full black hole solution (6) the helicity-0 model may or may not acquire its kinetic term due to nonzero $\mu$ and/or $b$.
} 
[3] C. de Rham, G. Gabadadze, D. Pirtskhalava, A. Tolley and I. Yavin, JHEP 1106028 (2011).

[4] S. Deser and R. I. Nepomechie, Annals Phys. 154, 396 (1984).

[5] A. Higuchi, Nucl. Phys. B282, 397 (1987).

[6] S. Deser and A. Waldron, Phys. Rev. Lett. 87, 031601 (2001).

[7] G. Gabadadze and A. Iglesias, JCAP 0802, 014 (2008).

[8] M. Blagojevic and B. Cvetkovic, JHEP 1103, 139 (2011).

[9] E. A. Bergshoeff, O. Hohm and P. K. Townsend, Phys. Rev. D79, 124042 (2009).

[10] J. Oliva, D. Tempo and R. Troncoso, JHEP 0907, 011 (2009).

[11] C. de Rham and G. Gabadadze, Phys. Rev. D82, 044020 (2010).

[12] C. de Rham, G. Gabadadze and A. J. Tolley, Phys. Rev. Lett. 106, 231101 (2011). 